\title{
Strategien zur Abdeckung der Fixkosten des Lebens: Empirische Untersuchungen zur Einkommensschaffung bedürftiger Teilbauern in Karangasem (Ostbali) ${ }^{1}$
}

\section{Einleitung}

Einkommenserhebungen von bedürftigen Personen in der Dritten Welt lassen oft außer acht, daß diese ihre Einkünfte in der Regel nicht bloß durch eine Beschäftigung, sondern durch eine Kombination von Erwerbstätigkeiten schaffen. Beschränkt man sich auf den im Zensus ausgewiesenen «Beruf» und das mit diesem verbundene Einkommen, fällt letzteres oft so tief aus, daß damit die Abdeckung des Existenzminimums und die Sicherstellung des Überlebens nicht gewährleistet wären. Überleben bedeutet im folgenden die Abdeckung der Fixkosten des Lebens unter einer stark schwankenden Arbeits-, Einkommens- und Ausgabensituation. Voraussetzung menschlichen Lebens ist der Verzehr bestimmter, für die Grundbedürfnisbefriedigung unabdingbarer materieller Güter und immaterieller Leistungen. Diesem entsprechen die Fixkosten des Lebens.

Wie der Ende der 70er Jahre von Bielefelder Entwicklungssoziologen (ELWERT, EVERS, SCHIEL, WONG u.a.m.) in die entwicklungspolitische Diskussion eingebrachte «Bielefelder Verflechtungsansatz» aufzeigt, können marginalisierte Bevölkerungsschichten ihr Überleben letztlich nur durch Produktionsformen sicherstellen, welche nicht ausschließlich auf dem "Lohnarbeit-Kapital-Verhältnis» beruhen. Dazu zählen beispielsweise unbezahlte Frauenarbeit im Haushalt, die Erstellung von Wohnraum durch Eigenleistung (Habitat-Reproduktion), der Anbau von Lebensmitteln für den Eigenbedarf usw. (EVERS 1978: 237). Die Bielefelder Forscher verweisen hierbei auf die Bedeutung der Verflechtung, die sowohl zwischen Subsistenzproduktion (Produktion für den Eigenbedarf) und Warenproduktion (Produktion für den Markt) als auch zwischen formellem und informellem Sektor ${ }^{2}$ zum Ausdruck kommt. Marginalisierte Schichten einer Gesellschaft können ihr Überleben letztlich nur durch konstantes strategisches Handeln sicherstellen. Das bedeutet, daß bei fehlendem Vermögen Überleben, Sicherheit und Stabilität der Vernetzung verschiedener ökonomischer Aktivitäten (Subsistenzproduktion, Warenproduktion, Lohnarbeiten) bedarf. Die Kombination der Aktivitäten muß dabei permanent der momentanen ökonomischen Situation angepaßt werden (ELWERT/WONG 1979: 227, ELWERT/EVERS/WILKENS 1983: 184).

Im vorliegenden Fallbeispiel werden die Strategien der Sicherstellung des Überlebens von fünf bedürftigen, als Teilbauern (engl.: share cropper; bal.: panyakap) tätigen Familien aus Ostbali untersucht und dargestellt. Bei dem in der indonesischen Landwirtschaft weit verbreiteten Teilbau (bagi hasil; sakap) handelt es sich, knapp formuliert, um einen Vertrag zwischen einem Landbesitzer bzw. Nutzungsberechtigten und einem Bewirtschafter zwecks Feldbestellung, wobei sich die Vertragsparteien den Input und den Ernteertrag (Output) gemäß einem vereinbarten Schlüssel teilen. Aufgrund der von den Respondentenfamilien bearbeiteten kleinen Landparzellen - zwischen 15 und 20 Aren Bewässerungsfelder (sawah) bzw. zwischen 35 und 95 Aren Trockenfelder (tegalan)und Abgabequoten von $1 / 2$ bis $2 / 3$ der landwirtschaftlichen Erträge kann aus dem Teilbau allein keine der Familien ihren Lebensunterhalt sicherstellen. Der vorliegende Artikel setzt sich daher zum Ziel, die inner- und außerbetrieblichen Einkommensschaffungen der Respondentenfamilien aufzuzeigen und damit einen Beitrag zum Verständnis der sozio-ökonomischen Situation eines bedeutenden Anteils der ostbalinesischen Bevölkerung zu leisten. Bei der Erfassung der Überlebensstrategien ist nebst individueller Betroffenheit - der gesellschaftlichen Vernetztheit durch gegenseitige wirtschaftliche, politische und sozio-kulturelle Beziehungen Rechnung zu tragen. Die das Überleben gewährleistenden Handlungsweisen der Untersuchungsfamilien müssen somit in ihrem sozio-kulturellen Kontext verstanden werden.

\section{Physischgeographische und anthropogeographische Grundlagen}

\subsection{Untersuchungsgebiet}

Die Erhebungen zur Fallstudie erfolgten im Herbst 1991 in zwei Dörfern der Regentschaft (kabupaten) Karangasem. Eine der Untersuchungsgemeinden, Padangkerta, liegt im Distrikt (kecamatan) Karangasem, die andere, Abang, im gleichnamigen Distrikt Abang. Die beiden Gemeinden unterscheiden sich vor allem in der vorherrschenden Landnutzung. In Padangkerta finden sich praktisch ausschließlich Bewässerungsfelder, während in dem von mir erfaßten Teil von Abang Trockenfelder dominieren.

Ralf Lünsmann, dipl. Geograph, Schürwiesstr. 11, 8855 Wangen 
Der in der Zone des rezenten Vulkanismus gelegene $\mathrm{ka}$ bupaten Karangasem weist ein hügeliges bis gebirgiges Relief auf. Kulminationspunkt ist der Vulkan Gunung Agung $(3142 \mathrm{~m})$. Das Klima ist monsunal geprägt. Abhängig von der Topographie, variieren die mittleren jährlichen Niederschlagsmengen beträchtlich. Sie belaufen sich in den mittleren Höhenlagen auf $1000 \mathrm{~mm}$ bis $2000 \mathrm{~mm}$, in höheren Lagen auf $2000 \mathrm{~mm}$ bis $3000 \mathrm{~mm}$. Die jährliche Durchschnittstemperatur auf Meereshöhe beträgt $25^{\circ}$ bis $27^{\circ}$, wobei die Schwankungen zwischen den Monatsmitteln lediglich $1,5^{\circ}$ bis $2,5^{\circ}$ ausmachen, was nicht zuletzt dem maritimen Einfluss zuzuschreiben ist. Mit zunehmender Höhe nehmen die Temperaturen ab, wobei das für die Tropen typische Tageszeitenklima bestehen bleibt (BUNDSCHU 1985: 11, LEEMANN 1979: 34 ff.). Rund $4 / 5$ der erwerbstätigen Bevölkerung des kabupaten Karangasem sind in der Landwirtschaft beschäftigt. Die Regentschaft ist touristisch wenig erschlossen und industriell noch kaum entwickelt. Von der im kabupaten Karangasem landwirtschaftlich genutzten Fläche von 83954 ha sind ungefähr $90 \%$ Trocken- und 10\% Bewässerungsfelder (KANTOR STATISTIK KARANGASEM 1991: 202). Bedingt durch den Bevölkerungszuwachs, die Realteilung des Bodens bei Erbschaftsübertragungen und den Mangel an ausseragrarischen Arbeitsplätzen herrschen in Karangasem (wie auf ganz Bali) Kleinbetriebe vor. Über $50 \%$ von ihnen weisen unter 0,5 ha Boden in Eigenbesitz, 20\% gar unter 0,2 ha auf. Ein Viertel aller Landwirte bearbeiten ausschließlich Land, das nicht ihr Eigentum ist (BUNDSCHU 1985: 19). Im Gemeindegebiet von Padangkerta wurden 1987 von insgesamt 1152 erfaßten Familienvorstehern deren 679 als Landwirte ausgewiesen; 507 davon waren Teilbauern! (KANTOR KEPALA DESA PADANGKERTA 1988: 3, 7). Andere Systeme der Landabtretung sind nicht erfaßt worden. Für das Dorf Abang liessen sich keine statistischen Daten beschaffen. Gemäß Angaben von Gewährsleuten kann jedoch von ähnlichen Größenordnungen wie in Padangkerta ausgegangen werden.

Auf künstlich bewässerten Feldern wird fast ausschließlich Reis angepflanzt. Nach der Einführung von Hochertragsvarietäten (High Yielding Varieties; $H Y V$ ) können zwei bis drei Ernten pro Jahr eingebracht werden, sofern die Wasserverhältnisse dies zulassen. Die wegen der Verwendung von zugekauften Saaten, Kunstdünger und Insektiziden kapitalintensive Kultivierung von HYVs übersteigt oft die Finanzkraft und das Know-how der kleinbäuerlichen Bevölkerung. Bei ganzjährig ausreichender Wasserzufuhr sind die Vegetationszyklen nicht ausschließlich vom Monsunregen abhängig. In einem solchen Fall finden sich oft nebeneinander Parzellen mit unterschiedlichem Vegetationsstand der Anbauprodukte. Aus klimatischen, edaphischen und bewässerungstechnischen Gründen wird oberhalb von rund $600 \mathrm{~m}$ kaum mehr Naßreis angepflanzt. Ist Reisanbau auch in tieferen Lagen nicht möglich, wird Trockenfeldbau (syn.: Regenfeldbau) betrieben. Hauptanbauprodukte sind in einem solchen Fall Mais und Kassava, d. h. dieselben Nutzpflanzen, die bei Wassermangel in der Trockenzeit auch auf sawah angebaut werden. Ferner werden Bohnen, Erdnüsse, Zwiebeln, verschiedene Knollengewächse und weitere Gemüse produziert. Von großer Bedeutung sind Dauerkulturen, insbesondere von Kokospalmen, Agrumen und anderen Früchten. Zunehmend wichtiger wird in den höheren Lagen die Produktion der cash crops Kaffee, Vanille und Gewürznelken (JAYASURIYA/NEHEN 1989: 336-339, BUNDSCHU 1985: 17).

\subsection{Untersuchungsfamilien}

Ausgangspunkt bei der Erfassung der bedürftigen Respondentenfamilien war die Erkenntnis, daß Bedürfnisse historisch gewachsen und durch die jeweilige sozio-ökonomische und politische Situation bzw. durch die Wahrnehmung dieser Situation geprägt sind (LEDERER/MAK. KENSEN 1975: 18-21). Nichtbefriedigte Bedürfnisse sind empirisch feststellbare Mangelzustände, die relativ zu bestimmten biologischen und gesellschaftlichen Normen definiert werden müssen (ENDERLE 1987: 17 f.).

Meine Untersuchungsfamilien weisen folgende Gemeinsamkeiten auf: (1) Alle Familienvorsteher sind Teilbauern (panyakap) ohne eigenen Landbesitz. (2) Der Naturallohn aus dem Teilbau reicht nicht aus, um den Eigenbedarf der Familie an Nahrungsmitteln zu decken. (3) Alle Familien leben in einfachen, auf dem bearbeiteten Land erstellten Feldhütten ( $k u b u$ ) und nicht - wie auf Bali üblich - in einem Familienhof im Dorf selber. Aus Gründen der Platzknappheit ist daher lediglich ein $\mathrm{Zu}$ sammenleben in Kernfamilien (Eltern und deren Kinder) möglich. Sämtliche Respondenten sind - zufälligerweise - verarmte Angehörige des balinesischen Adels (triwangsa). Die Kinder - zwischen zwei und fünf pro Familie - sind alle jünger als elf Jahre. Die beiden in Padangkerta erhobenen Familien A und B bearbeiten als share croppers 15 bzw. 20 Aren sawah. Die drei in Abang untersuchten Familien C, D und E bewirtschaften 95, 35 bzw. 65 Aren tegalan im Teilbau. Sämtliche Respondenten halten zudem Rinder, Schweine und Geflügel. Ein Großteil der Tiere ist jedoch nicht Eigenbesitz, sondern wird als Pensionsvieh gehalten. Bei der als Analogie zum Teilbau zu betrachtenden Vertragsviehhaltung (ngadas) nimmt der Halter Tiere in Pflege, welche ihm, etwa im Falle von Rindern, als Arbeitstiere zur Verfügung stehen (RÖLL/LEEMANN 1987: 84f.). Beim Verkauf von Vieh durch die Besitzer steht den Respondenten die Hälfte des Verkaufserlöses zu. Darüber hinaus haben sie Anrecht auf einen bestimmten Anteil an Jungtieren aus der Nachzucht.

\section{Fixkosten des Lebens}

In diesem Kapitel sollen die jährlichen Fixkosten des Lebens der Untersuchungsfamilien ermittelt werden. Darunter ist - wie erwähnt - der Verzehr der zur Grundbedürfnisbefriedigung unabdingbaren, materiellen Güter und immateriellen Leistungen zu verstehen. Im folgen- 


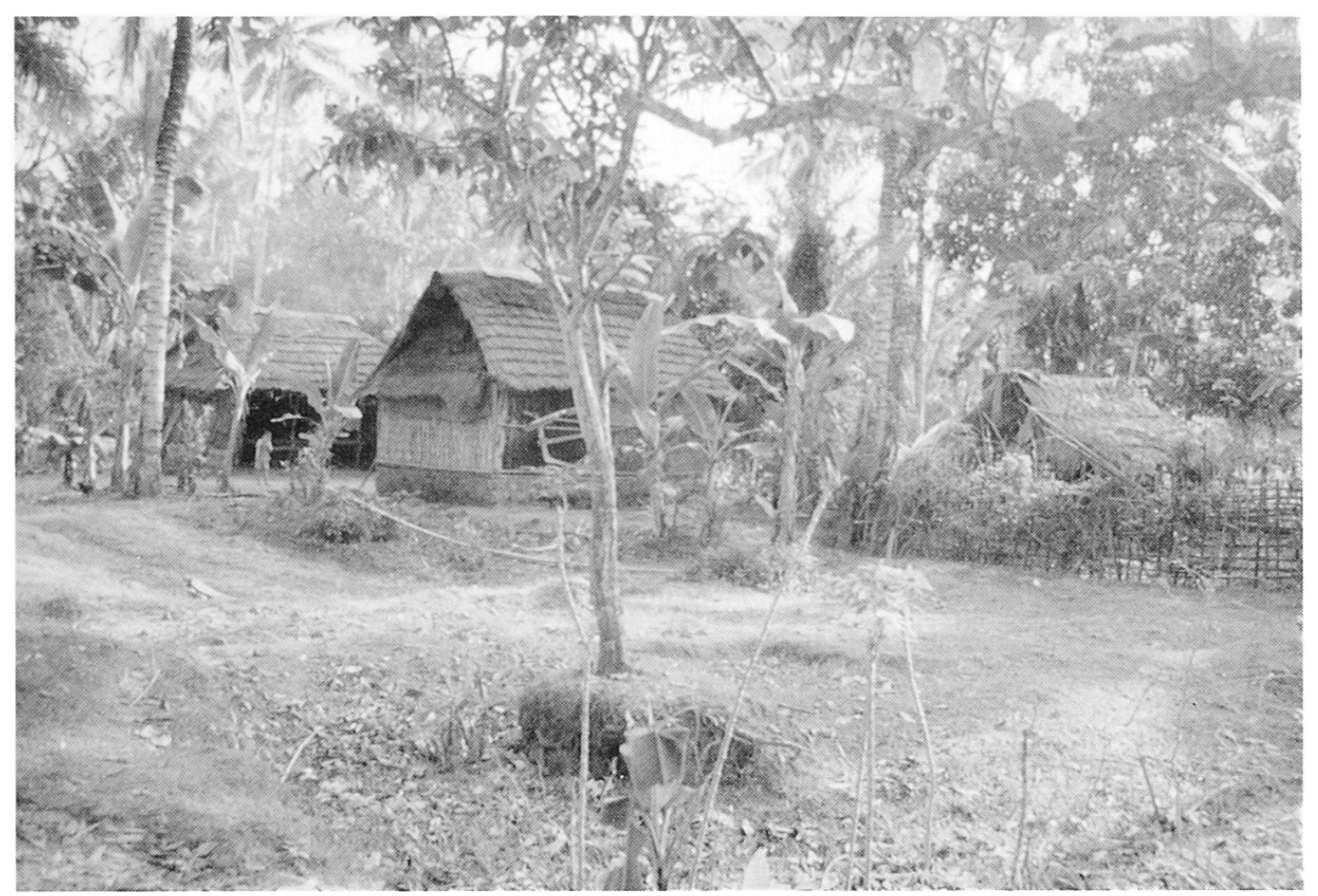

Abb. 1 Kubu der Familie A in Padangkerta.

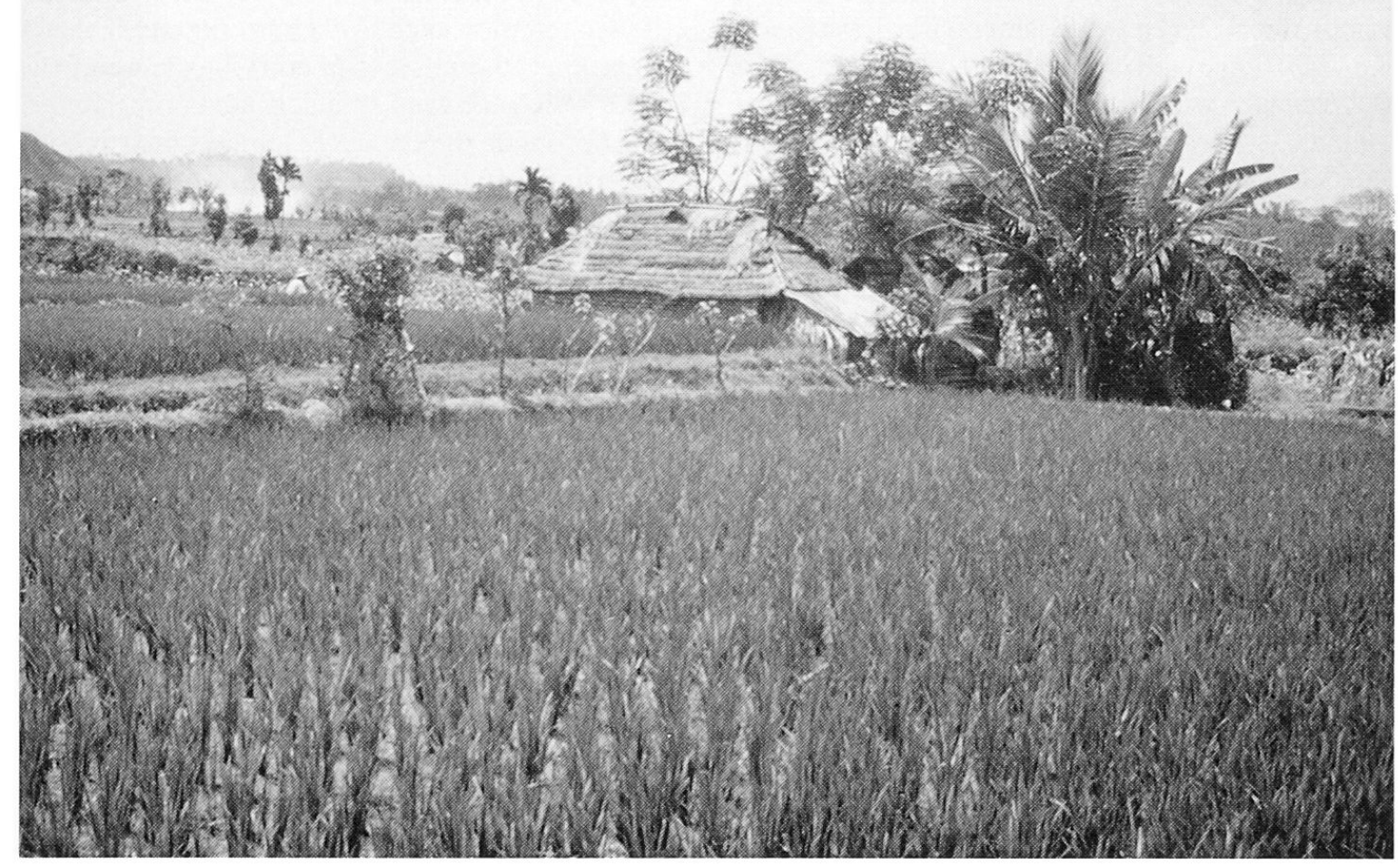

Abb. 2 Kubu der Familie C in Abang. 
den werden lediglich die mir von den Familien selbst mitgeteilten und von ihnen als notwendig erachteten (materiellen) Grundbedürfnisse aufgelistet und quantifiziert. Als Rechnungseinheit dient die Landeswährung Rupiah (im Herbst 1991 belief sich der Wechselkurs von SFr. 1.auf Rp. 1200.- bis Rp. 1400.-). Die minimalen Lebensunterhaltskosten liegen bei sämtlichen Familien in ähnlicher Größenordnung, weshalb auf eine differenziertere Betrachtung der einzelnen Haushalte verzichtet wird. Die nachstehend angeführten Preisangaben sind lediglich als Größenordnung zu betrachten und sollen dazu dienen, dem Leser Einblick in die wirtschaftliche Situation der Familien zu gewähren.

Obwohl alle Untersuchungsfamilien sich als Teilbauern betätigen, ist es keiner möglich, ihren gesamten Nahrungsmittelbedarf aus Eigenerzeugung zu decken. Sie sind darauf angewiesen, zusätzlich Lebensmittel über den Markt zu beschaffen. Die Speisepläne sämtlicher Familien gleichen sich. Haupt- bzw. Leitnahrungsmittel ("cultural superfood") ist Reis. Keine der Familien ist willens, Reis vollständig durch preisgünstigere, aber soziokulturell minderbewertete Nahrungsmittel wie beispielsweise Mais oder Süßkartoffeln zu substituieren. Normalmahlzeiten umfassen gekochten Reis (nasi putih), Gemüse und geröstete Erdnüsse, gelegentlich ergänzt durch etwas getrockneten Fisch, Mais oder Süßkartoffeln. Zum Essen werden in der Regel Salz, cabai (Capsicum annuum) und geraspeltes Fruchtfleisch der Kokosnuß gereicht. An Festtagen und Zeremonien fällt die Zusammensetzung der Nahrung reichhaltiger aus und umfaßt dann meist auch Fleisch und Eier. Der Bedarf an enthülstem Reis (beras) liegt gemäß Auskunft der Respondenten bei rund $180 \mathrm{~kg}$ pro Kopf und Jahr. Eine fünfköpfige Familie benötigt somit jährlich $912,5 \mathrm{~kg}$ beras. Bei einem Preis von Rp. 650.- pro Kilogramm im Oktober 1991 entsprechen die 912,5 kg beras einem Marktwert von rund Rp. 600 000.- (Rp. 593 125.-), das sind etwa SFr. 450.- . Beilagen wie Gemüse, Süßkartoffeln und Erdnüsse stehen allen Respondenten weitgehend aus Eigenanbau zur Verfügung. Zugekauft werden müssen jedoch jährlich für rund Rp. 60 000.- Kaffee, für Rp. 28 000.Zucker, für Rp. 3600.- Salz und Rp. 15 000.- Diverses wie Öl, Farben zur Kolorierung zeremonieller Opferspeisen usw. Zusammenfassend ergibt sich für eine fünfköpfige Respondentenfamilie ein jährlicher Aufwand für Lebensmittel von rund Rp. 706 600.- (ca. SFr. 540.-), welcher mit Ausnahme des als Naturallohn aus dem Teilbau erwirtschafteten Reises überwiegend über den Markt beschafft werden muß.

Die jährlichen Fixkosten für Unterkunft, Hausrat, Kleider usw. sind bescheiden. Die an und für sich nur provisorisch errichteten Feldhütten, welche die Respondenten jedoch permanent bewohnen, sind mit Familien- und Nachbarschaftshilfe (tolong-menolong) in Eigenleistung erbaut worden. Dabei wird vor allem auf lokale Ressourcen, so Bambus, Kokospalmblätter, Reisstroh und luftgetrocknete Lehmziegel, zurückgegriffen. Gewisse Arbeitsgeräte wie Sicheln, Messer, Hacken, die nicht selber hergestellt werden können und einem hohen Verschleiß unterliegen, müssen oft jedes Jahr neu angeschafft werden. Weitere Ausgaben erwachsen den Familien aus der Beschaffung von Lampenöl, Seife, Kleidern (u. a. Schuluniformen für ihre Kinder), Schulmaterialien usw. Pro Jahr muß eine Respondentenfamilie mit zwei Kindern im schulfähigen Alter für die genannten Bedarfsgüter im Minimum Rp. 71 000.- (rund SFr. 60.-) aufwenden. Ohne Berücksichtigung der für den Eigenbedarf angebauten und nicht unter die Teilbauvereinbarung fallenden Nahrungsmittel sowie der in Subsistenzproduktion hergestellten Bedarfsgüter müssen die Familien somit mit jährlichen Fixkosten für Nahrungsmittel und andere Waren in der Größenordnung von Rp. 778 000.- (ca. SFr. 600.- ) rechnen.

\section{Schwankende Einkommens- und Ausgabensituation}

Die Abdeckung der in Kap. 3 aufgezeigten Fixkosten des Lebens erfolgt bei den Untersuchungsfamilien unter ungünstigem Einfluß stark schwankender Erwerbsmöglichkeiten und Einkommen, aber auch variierender Ausgaben. Das Einkommen der bäuerlichen Bevölkerungsschicht hängt in erster Linie vom Vegetationszyklus ab. der in monsunalen Gebieten ausgesprochen saisonal geprägt ist. Dies führt zu einem fluktuierenden Arbeitsanfall und - als Folge davon - zu langen Phasen der Unterbeschäftigung von Familien, die auf Erwerbstätigkeiten in der Landwirtschaft angewiesen sind (BLANCKENBURG 1986: 60). Aber auch Umwelteinflüsse wie Variabilität der Niederschläge, Schädlingsbefall usw. verursachen große Unsicherheiten und Schwankungen in der bäuerlichen Einkommensschaffung. Zu beachten ist, daß normenverpflichtetes Handeln in gewissen Situationen (wie z. B. bei zeremoniellen Anlässen) beträchtliche Ausgaben bedingt und einen großen Arbeitseinsatz verlangt. Solche Aufwendungen sind deshalb unumgänglich, weil so die Verbundenheit mit der Gemeinschaft unter Beweis gestellt und Sozialprestige gewonnen werden kann. Kostspielige Zeremonien - v. a. Kremationen - haben nicht selten lebenslange Verschuldung oder Veräußerung von eventuell vorhandenem Landbesitz zur Folge (LEEMANN 1979: 85 ff.). Aber auch Erkrankungen, Unfälle oder hohe Verluste bei Geld- und Wettspielen (Hahnenkämpfe, Domino usw.) können zu unvorhergesehenen Ausgaben führen bzw. zur Verschuldung beitragen.

\section{Abdeckung der Fixkosten}

\subsection{Einkommen aus dem Teilbau}

Da alle befragten Familienvorsteher als ihre Hauptbeschäftigung die des Teilbauern nennen, soll zunächst das Einkommen aus dem share cropping ermittelt werden. Bei den örtlichen Teilbaukontrakten handelt es sich um eigentliche Arbeitsverträge zwischen Landbesitzern bzw. Nutzungsberechtigten und Landbearbeitern ( $p a-$ 
nyakap). Für das eingeräumte Bearbeitungsrecht hat der begünstigte panyakap einen vertraglich vereinbarten Anteil des Ernteertrages an den Arbeitgeber abzutreten. Der Rest gilt als Abgeltung für die Mühewaltung des Teilbauern (RÖLL/LEEMANN 1987: 186). Obwohl die Rechtsnatur der bagi hasil-Verträge nicht eindeutig bestimmt ist, verwehren sich Adat rechtsgelehrte gegen eine Gleichsetzung von Pacht mit Teilbau. Objekte des Teilbauvertrages sind nämlich nicht Grund und Boden, sondern Arbeit und Agrarprodukte. Teilbauverträge - zumindest in Indonesien - sind daher eher mit einer Feldbestellung im Naturallohnsystem als mit einer Grundstücktransaktion verwandt. Auch trägt der Landeigentümer bei Teilbauvereinbarungen - im Gegensatz zur Pacht - das wirtschaftliche Risiko mit. Auffällig ist, daß bei meinen Respondenten das Beziehungsgefüge zwischen Teilbauer und Nutzungsberechtigtem bzw. Landbesitzer über die in der Pacht üblichen Formen hinausgeht und Ähnlichkeiten zu hierarchischen Familienstrukturen aufweist (vgl. auch KAELIG 1986: 18 f., 32): Der Grundeigentümer nimmt de facto die Position eines ranghöheren Familienmitgliedes ein und fühlt sich daher auch verpflichtet, seinen panyakap in sozialen Belangen beizustehen.

Obwohl bagi hasil-Verträge für einen Teilbereich der Landwirtschaft, nämlich den Anbau von Nutzpflanzen mit kurzer Vegetationsdauer, durch die staatliche Agrargesetzgebung geregelt sind, läßt die Implementierung der Gesetzesbestimmungen in vielen Fällen zu wünschen übrig. Um lokalen Gegebenheiten Rechnung zu tragen, liegt es zudem in der Befugnisgewalt des bupati (Vorsteher einer Regentschaft), gewisse, von den Richtlinien abweichende Verteilerschlüssel festzusetzen. Als Regel bei der Aufteilung gilt, daß bei Naßreis $1 / 2$, bei palawija und Trockenfeldbauprodukten $2 / 3$ des Ernteanteils dem Bewirtschafter zustehen (KAEHLIG 1986: 229, BACKHAUS 1990: 129).

Sawah bestellende Respondenten in Padangkerta erhalten als share croppers $1 / 3$ des Reisertrages (ind.: bagi tiga; bal.: nelon). In der auf den Reisanbau folgenden Vegetationsperiode, in welcher vorwiegend Mais sowie Erdnüsse, Bohnen und Süßkartoffeln angebaut werden, wird die Ernte im Verhältnis 1:1 aufgeteilt (ind.: bagi dua; bal.: nandu). In Abang hingegen werden alle agraren Erzeugnisse - auch Reis und Kokosnüsse - hälftig geteilt.

Der Marktwert des den Respondenten aus dem Teilbau zufallenden Naturallohnes liegt dabei je nach Familie zwischen Rp. $126000 .^{-}$(ca. SFr. 100.-) und Rp. 337 000.- (ca. SFr. 260.-). Dies entspricht 16\% bis $43 \%$ der monetär erfaßbaren Lebensunterhaltskosten von rund Rp. 778 000.-- Zur Illustration sei im folgenden das Teilbaueinkommen eines Respondenten in Padangkerta aufgeschlüsselt: Respondent A kann mit seinen Familienangehörigen auf 15 Aren sawah im jährlichen Durchschnitt $450 \mathrm{~kg}$ beras einbringen. Der Reis wird im mederep-Verfahren geerntet, bei dem die als Erntearbeiter beteiligten Personen einen relativen Anteil des von ihnen geschnittenen Reises als Arbeitslohn erhalten. Grundlage der traditionellen Arbeitsorganisation und überlieferter Praktiken ist die adatrechtliche Verpflichtung eines jeden Landbesitzers bzw. Nutzungsberechtigten (auch eines Pächters oder Teilbauern), Dorfbewohner an der Ernte teilnehmen zu lassen. Diese können dafür in der Regel 1/11 (nyolasin) des individuell geschnittenen gabah (nichtenthülster Reis) beanspruchen. Der zur Ernte einladende Landwirt kann seinerseits erwarten, andernorts wiederum zu Schnitterarbeiten aufgeboten zu werden. So gewährleistet dieses traditionelle Sozialsystem, daß zumindest während der Erntezeiten keine Familie unter Nahrungsmittelmangel zu leiden hat (RÖLL/ LEEMANN 1987: 225). Rund $30 \mathrm{~kg}$ beras muß Respondent A als Erntelohn abführen. Von den restlichen $420 \mathrm{~kg}$ beras sind $2 / 3$ dem Landeigentümer abzutreten. Der Familie stehen somit lediglich $140 \mathrm{~kg}$ beras (Geldwert 1991: Rp. 91 000.- ) zu, d. h. knapp $1 / 6$ des in Kap. 3 für eine fünfköpfige Familie ermittelten jährlichen Reisbedarfs von rund $900 \mathrm{~kg}$. Des weiteren verbleiben der Familie nach erfolgter Ernteteilung jährlich rund 400 Maiskolben (Marktwert: Rp. $10000 .^{-}$) und rund $50 \mathrm{~kg}$ Erdnüsse (Rp. 25 000.-) sowie geringe, nichtteilungspflichtige Mengen an Süßkartoffeln und Gemüse, die ausschließlich dem Eigenverzehr dienen. Zusammenfassend kann Respondent A aus seiner Tätigkeit als Teilbauer jährlich ein Naturaleinkommen im Marktwert von rund Rp. 126 000.- (entsprechend SFr. 100.-) erwirtschaften.

Angesichts der oben angeführten, niedrigen Einkommen aus dem Teilbau ist allerdings zu berücksichtigen, daß alle Familien von den Landeigentümern die Möglichkeit zugestanden bekommen haben, in bescheidenem Ausmaß nicht unter die Teilungspflicht fallende Nährpflanzen für den Eigengebrauch anzubauen. Zudem erlaubt der Zugang zu Land auf bagi hasil-Basis die Errichtung einer bescheidenen Unterkunft (kubu) und die Haltung von Vieh und Geflügel. Auch hat der Teilbauer im Gegensatz zum eigentlichen Tagelöhner eine unternehmerische Funktion und kann seine Arbeit weitgehend unabhängig und ohne Kontrolle verrichten. Er befindet sich daher in einer sozio-ökonomisch eindeutig besseren Position als der landlose Tagelöhner (vgl. KAELIG 1986: 19).

\subsection{Einkommen aus Vieh- und Geflügelhaltung}

Neben Erträgen aus dem Teilbau stellen Vieh und Geflügel eine der wesentlichsten Einkommensquellen der Respondentenfamilien dar. Der Verkauf von Tieren bietet oft die einzige Möglichkeit, kurzfristig zu Bargeld zu gelangen und damit temporäre finanzielle Engpässe zu überbrücken. Alle befragten Familien halten balinesische Rinder, Schweine und Hühner bzw. (Kampf-)Hähne, Familie A zeitweise auch Enten. Über die Tiere kann jedoch nur dann frei verfügt werden, wenn es sich dabei um Eigenbesitz handelt. Ein Großteil des Viehbestandes meist Rinder und Schweine - werden aber lediglich in Pflege (ngadas) gehalten.

Aufgrund der starken Fluktuationen beim Tierbestand ist der Einkommensanteil der Respondenten aus der Vieh- und Geflügelhaltung nicht genau zu beziffern. 
Kann eine Familie bespielsweise pro Jahr ein bis zwei Schweine aus Eigenbesitz zu Rp. 70 000.- und 30 Hühner zu Rp. 2000.- verkaufen, entspricht dies einem Zusatzeinkommen von Rp. 130 000.- bis Rp. 200000.(entsprechend SFr. 100.- bis SFr. 150.-), welches in der Größenordnung des Betrages liegt, den die Bewirtschaftung des Bodens im Teilbau einbringt.

\subsection{Einkommen aus Nebenerwerbstätigkeiten}

Wie oben belegt, können die Respondentenfamilien mit dem Einkommen, das aus ihrer Tätigkeit als Teilbauer (panyakap) und als Vertragstierhalter (pangadas) bzw. Eigentümer von Vieh und Geflügel resultiert, nur einen Teil ihrer Lebensunterhaltskosten bestreiten. Die Familien versuchen daher, durch Intensivierung der Subsistenzproduktion (vor allem im Bereich des Hausrates) die monetären Fixkosten so tief wie möglich zu halten. Sie betrachten dabei die Subsistenzproduktion größtenteils als nichtkostenverursachend (vgl. auch ELWERT/ WONG 1979: 263 f.).

Obwohl der gebrauchswertorientierten Produktion eine zentrale Bedeutung zukommt, ist es den Familien nicht möglich, ihre Existenz ohne eine Kombination von mehreren, meist informellen Nebenerwerbstätigkeiten sicherzustellen. Der Zugang zu Lohnarbeit ist jedoch gemäß EVERS (1978: 139) erst durch einen Input von Subsistenzproduktion möglich, da Löhne oft so tief sind, daß diese durch Subsistenzproduktion, insbesondere in Form unbezahlter Frauenarbeit, subventioniert werden müssen.

Die durch Teilbau, Tierhaltung und Subsistenzproduktion nicht gedeckten Lebensunterhaltskosten müssen durch außerbetriebliche Einnahmen beglichen werden, welche mit dazu beitragen, Produkte und Dienstleistungen kaufen zu können, die nur über den Markt erhältlich sind. In Kap. 3 wurden die von der Subistenzproduktion subventionierten, minimalen Lebensunterhaltskosten für eine fünfköpfige Familie auf rund Rp. 778000.- beziffert. Hiervon können rund die Hälfte bis zwei Drittel durch innerbetriebliche Tätigkeiten, d. h. durch Teilbau und Tierhaltung, erarbeitet werden. Um die gesamten Fixkosten abzudecken, müssen somit durch Nebenerwerbstätigkeiten je nach Familie zusätzlich rund Rp. $250000 .^{-}$bis Rp. $380000 .^{-}$(entsprechend SFr. 200.- bis SFr. 300.-) eingenommen werden. Das unzureichende Arbeitsplatzangebot zwingt die Respondenten zu einer "occupational multiplicity», d. h. zum Zuerwerb durch in der Regel verschiedene, sich gerade anbietende Arbeitsmöglichkeiten. Wenn immer möglich, gehen share croppers einem agraren Nebenerwerb nach, der ihrem Erfahrungshorizont als Bauer entspricht (RÖLL/LEEMANN 1987: 220).

In Padangkerta verdingen sich Angehörige der beiden Respondentenfamilien - insbesondere die Ehefrauen vorzugsweise als Tagelöhner in der Landwirtschaft. Höchste Tagesverdienste werden bei Erntearbeiten im mederep-Verfahren erzielt (vgl. RÖLL/LEEMANN 1987: 224), nämlich 3 bis $4 \mathrm{~kg}$ beras im Gegenwert von rund
Rp. 2000.- bis Rp. 2600.-. Kann ein Familienmitglied während 30 bis 50 Tagen pro Jahr Erntearbeiten im mederep-Verfahren nachgehen, führt dies zu einem jährlichen Zusatzeinkommen von Rp. $60000 .^{-}$bis Rp. 130 000.- .

Wichtigste Nebenerwerbstätigkeit der Respondenten in Abang ist das Graben nach andesitischen Gesteinsbrokken auf den von ihnen bearbeiteten Trockenfeldparzellen. Die zu Tage geförderten Steine werden vorerst mit Vorschlaghammer und Brecheisen zerkleinert und dann an Bauunternehmen verkauft. Ein kräftiger Arbeiter kann in zwei bis drei Tagen eine Lastwagenladung (ca. 3,5 $\mathrm{m}^{3}$ ) Steine zubereiten, die mit Rp. 6500.- vergütet wird. Andere Nebeneinkommen schaffen die Respondenten aus Abang mit der Herstellung von Flechtwaren, dem Verkauf von Agrarprodukten sowie durch Wahrnehmung kurzfristiger öffentlicher oder privater Arbeitsaufträge (z. B. Tempelrenovationen, Straßenbau). Oft müssen für den Landeigentümer außeragrarische Dienstleistungen - z. B. bei der Vorbereitung von Zeremonien erbracht werden. Dafür werden die panyakap nicht entlöhnt, sondern nur verpflegt. Gelegentlich erhalten sie zusätzlich Nahrungsmittel für ihre Familien.

Da die für landwirtschaftliche Tätigkeiten gewährten Löhne bei gleicher Arbeitszeit häufig das Einkommen aus Kleingewerbe, Kleinhandel usw. übertreffen, geben die Respondenten in Zeiten agrarischer Arbeitsspitzen ihre oft nur als komplementär betrachteten außeragrarischen Nebenerwerbstätigkeiten - wie beispielsweise das Korbflechten - auf, um einer besser entlöhnten Beschäftigung in der Landwirtschaft (v.a. als Erntearbeiter) nachzugehen (RÖLL/LEEMANN 1987: 220).

\section{Zusammenfassung}

Die Deckung des Minimaleinkommens erfolgt bei meinen Respondentenfamilien unter dem ungünstigen Einfluß eines stark schwankenden Zugangs zu Arbeit, eines unregelmäßigen Einkommens aus landwirtschaftlichen Tätigkeiten und variierender Ausgaben. Der saisonal geprägte Arbeitsanfall in der Landwirtschaft führt zu langen Phasen von Unterbeschäftigung und periodischen Erwerbsausfällen. Gerade die vermögenslosen, durchwegs unversicherten Teilbauern können auch durch Umwelteinflüsse massiv in ihrer Existenzgrundlage getroffen werden. Zeremonien, insbesondere rites de passage und Feiern für Verstorbene, verursachen bedeutende Ausgaben und führen oft zur Verschuldung. Aber auch Unfall und Krankheiten stellen nicht zu unterschätzende Risikofaktoren dar.

Um ihren Lebensunterhalt zu sichern, müssen die Respondentenfamilien die erwähnten Risikofaktoren in Betracht ziehen und spezifisch angepaßte Überlebensstrategien entwickeln. Gewisse Sicherheit gewährleisten insbesondere die Subsistenzproduktion und eine «occupational multiplicity». Produktionsformen wie Subsistenzproduktion, Teilbau (sakap; bagi hasil) und der Zugang zu 
Erntearbeiten im mederep-Verfahren lassen sich als wichtige Bestandteile einer in die Gesellschaft eingebetteten Gesamtwirtschaft erkennen. Sie ermöglichen letztlich auch die Reproduktion sozialer Formationen. Die Expansion von Kapitalismus und Warenproduktion und die damit einhergehende «Modernisierung» (z. B. Grüne Revolution) können zur Desintegration der familiären Ökonomie führen und eine Vernachlässigung sozialer Verpflichtungen und traditioneller Formen gegenseitiger Hilfe und kollektiver Arbeit zur Folge haben (ELWERT/ wONG 1979: 260 f.) Politische und ökonomische Bedingungen lassen sich nicht losgelöst von der sozialen und kulturellen Dimension einer Gesellschaft betrachten; sie bedingen sich gegenseitig. Durch eine unreflektierte Kommerzialisierung traditionell überlieferter agrarer Wirtschaftssysteme und die meist damit verbundene Hinwendung zu «rationelleren» Bewirtschaftungsformen vergrößert sich die Gefahr der Bildung eines land- und arbeitslosen Proletariats mit allen damit verbundenen wirtschaftlichen, sozialen und politischen Konsequenzen.

\section{Anmerkungen}

' Der Artikel basiert auf der Diplomarbeit des Autors: "Überlebensstrategien bedürftiger Teilbauernfamilien - Fallstudie aus Karangasem (Ostbali/Indonesien)" (1992), ausgeführt am Geographischen Institut der Universität Zürich. Die empirischen Erhebungen (u. a. mit teilnehmender Beobachtung, qualitativen Interviews und standardisiertem Fragebogen) wurden im Herbst 1991, während eines dreimonatigen Feldaufenthalts bei fünf Respondentenfamilien, durchgeführt. Die Konzipierung der Arbeit ("Fixkostenansatz") basiert auf grundlegenden Anregungen von Dr. R. Steppacher (Binningen) und Prof. Dr. A. Leemann (Zürich), denen ich an dieser Stelle meinen Dank ausspreche.

2 Der meist unpräzis verwendete Begriff “informeller Sektor” ist eine deskriptiv und nicht analytisch zu verwendende Bezeichnung für einen Bereich der Warenökonomie, die von den zuständigen staatlichen Behörden nicht als formelle ökonomische Aktivität erfaßt wird. Im Gegensatz dazu steht der formelle Sektor, in dem Unternehmen offiziell auftreten, im Handelsregister eingetragen sind und unter behördlicher Kontrolle stehen (vgl. dazu SCHIEL 1987:94).

\section{Literatur}

BACKHAUS, N. (1990): Aspekte der indonesischen Agrarverfassung (UUPA) und ihre Folgewirkungen, Diplomarbeit am Geographischen Institut der Universität Zürich.

BLANCKENBURG, P. von (1986): Welternährung: Gegenwartsprobleme und Strategien für die Zukunft, München.

BUNDSCHU, I. (1985): Probleme der agraren Grundbesitzverfassung auf Bali/Indonesien, Hamburg.

ELWERT, G./WONG, D. (1979): Thesen zum Verhältnis von Subsistenzproduktion und Warenproduktion in der Dritten Welt. In: Arbeitsgruppe Bielefelder Entwicklungssoziologen (Hg.), Subsistenzproduktion und Akkumulation, Bielefeld, 255-278.

ELWERT, G./EVERS, H.-D./WILKENS, W. (1983): Die Suche nach Sicherheit: Kombinierte Produktionsformen im sogenannten informellen Sektor. In: Zeitschrift für Soziologie, Nr. 4. 281-296

ENDERLE, G. (1987): Sicherung des Existenzminimums im nationalen und internationalen Kontext, Bern.

EVERS, H.-D. [et al.]. (1978): Subsistenzproduktion und Akkumulation, 2. Aufl., Frankfurt a. M.

JAYASURIYA, S. /NEHEN, I K. (1989): Bali: Ecomomic Growth and Tourism. In: Hill, H. (Hg.), Unity and Diversity: Regional Economic Development in Indonesia since 1970, Singapore, 330-348.

KAELIG, C.-B. (1986): Gesellschaft in Indonesien: autonome und nationale Gesellschaftsformen, Hamburg

KANTORKEPALA DESA PADANGKERTA(1988): Daftar isian potensi desa Padangkerta, Padangkerta/Bali (Manuskript).

KANTOR STATISTIK KARANGASEM (1991): Karangasem dalam angka, Amlapura/Bali.

LEDERER, K./MACKENSEN, R. (1975): Gesellschaftliche Bedürfnislagen, Göttingen.

LEEMANN, A. (1979): Bali: Insel der Götter, Innsbruck, Frankfurt a. M.

RÖLL, W./LEEMANN, A. (1987): Agrarprobleme auf Lombok: Untersuchungen zur Wirtschafts- und Sozialstruktur in Nusa Tenggara Barat, Indonesien, Hamburg.

SCHIEL, T. (1987): Suche nach Sicherheit und Sehnsucht nach Geborgenheit: "Dualwirtschaft" und "informeller Sektor" als Phänomen und Fiktion. In: Zeitschrift für Soziologie, Nr. 2, 92-105. 